\title{
Dinámica fluviométrica y limnológica de un cuerpo superficial de agua asociado a un prado húmedo de los Andes del norte-centro de Chile
}

\author{
Stream-flow and limnological dynamics of a surface body-water associated \\ with a wet-pasture of the Andes of north-central Chile \\ Jorge Cepeda-Pizarro ${ }^{1 *}$, Alfonso Antonio Armijo León ${ }^{2}$
}

\begin{abstract}
RESUMEN
A partir de una base de datos que cubrió 18 años, se analizó el comportamiento de algunos parámetros limnéticos de un cuerpo superficial de agua presente en una vega ubicada en la alta montaña de una hoya hidrográfica de los Andes del norte-centro de Chile. Se documenta la dinámica fluviométrica y la variación temporal de diversos parámetros físicos e hidroquímicos. El sistema se caracterizó por una elevada variabilidad temporal (tanto anual como mensual) de los parámetros estudiados. Esta variabilidad impidió detectar patrones temporales. El caudal más bajo mostró una clara correspondencia con la precipitación observada, no así el caudal más alto. Las características hidroquímicas del cuerpo de agua son consistentes con las características geológicas del área de estudio.
\end{abstract}

Palabras clave: limnología andina, Andes desérticos, ecosistemas de montaña, vegas andinas, ríos andinos.

\section{ABSTRACT}

By using an 18-years database, it was analyzed the behavior of a series of limnological parameters of a surface water body found in a wet-pasture located in the high mountain area of a river basin of north-central Chile. It is documented its stream-flow dynamics and the temporal variation of diverse physical and hydrochemical parameters. The system is characterized by a high temporal variability (both annually and monthly) of the parameters studied. This variability prevented to observe temporal patterns. Unlike the highest stream flow, the lowest flow showed a clear correspondence with the observed precipitation. The hydrochemical characteristics of the water body are consistent with the geological substrate of the study area.

Key words: Andean limnology, desert Andes, alpine ecosystems, high-land wet-pastures, Andean streams.

\section{Introducción}

En aquellos lugares de los Andes del nortecentro de Chile donde las quebradas se ensanchan y la rapidez del flujo de las aguas freáticas y superficiales disminuye, se forman las vegas andinas (Cooper et al., 2010; Squeo et al., 2006). El sustrato geológico por donde fluyen las aguas que mantienen las condiciones hídricas de estos sistemas y las características climáticas determinan las condiciones físicas y químicas de estas como reservorios de agua, recursos forrajeros, vida silvestre y diversidad biológica (Ginocchio et al., 2008). En este trabajo se describe y caracteriza la dinámica fluviométrica, hidrofísica e hidroquímica del estero Tambo, cuerpo de agua que forma parte de la vega Tambo-Puquíos, unidad de paisaje de la alta montaña andina del norte-centro de Chile. Específicamente, los objetivos del trabajo fueron: 1) caracterizar la dinámica fluviométrica del estero Tambo y 2) documentar la variabilidad altitudinal y temporal de algunos de sus parámetros físicos y químicos.

\section{Materiales y Métodos}

\section{Sitio de estudio}

Las características climáticas del área de estudio se describen en Cepeda y Novoa (2006). El trabajo

\footnotetext{
1 Departamento de Biología. Universidad de La Serena, Chile.

2 Departamento de Ingeniería de Procesos. Universidad de Las Palmas de Gran Canaria, Las Palmas de Gran Canaria, España.

* Autor para correspondencia: jcepeda@userena.cl
} 
se realizó en la vega "Tambo-Puquíos" (VTP en adelante), la que forma parte de la microcuenca del río del Toro, en la alta montaña de la hoya hidrográfica del río Elqui (Región de Coquimbo, Chile). Descripciones detalladas de esta microcuenca y de la VTP se encuentran en Cepeda-Pizarro (2013). El estero de interés, estero Tambo (ET en adelante), corre por el borde oeste de VTP.

\section{Parámetros examinados}

La mayoría de los parámetros considerados en el estudio están incluidos en las normas secundarias de calidad ambiental para aguas continentales y superficiales chilenas, detalladas en CONAMA (2000). La información base fue proporcionada por la Oficina de Asuntos Ambientales de la ex Compañía Minera El Indio (Barrick, Chile), cuyo acceso fue recientemente liberado de la condición de uso restringido y privado. Para la descripción y caracterización fluviométrica de ET se emplearon registros mensuales del período 1983-1999, obtenidos de una estación fluviométrica estándar ubicada a 3.940 m s n m. Para la descripción y caracterización de la variabilidad temporal de los parámetros físicos e hidroquímicos se usó información del período 19821999, obtenida a los $3.940 \mathrm{~m} \mathrm{~s} \mathrm{n} \mathrm{m}$. Para este fin se consideraron los siguientes parámetros, codificados

Tabla 1. Promedio mensual, estacional y anual del caudal superficial (1/s) del estero Tambo. Altitud: $3.940 \mathrm{~m} \mathrm{~s} \mathrm{n} \mathrm{m.}$ Serie de tiempo: 1983-1999.

\begin{tabular}{llc}
\hline \multicolumn{1}{c}{ Parámetro } & Media \pm DE \\
\hline 1. Mes & & \\
& Enero & $22,4 \pm 322,2$ \\
& Febrero & $86,4 \pm 75,0$ \\
& Marzo & $72,1 \pm 65,3$ \\
Abril & $61,9 \pm 30,1$ \\
Mayo & $59,9 \pm 25,8$ \\
& Junio & $49,1 \pm 15,6$ \\
& Julio & $48,4 \pm 18,1$ \\
& Agosto & $62,7 \pm 26,0$ \\
& Septiembre & $89,7 \pm 48,0$ \\
& Octubre & $84,3 \pm 52,0$ \\
& Noviembre & $151,7 \pm 212,3$ \\
& Diciembre & $210,5 \pm 219,8$ \\
2. Estación & $127,7 \pm 145,6$ \\
& Verano & $56,0 \pm 7,4$ \\
& Otoño & $67,0 \pm 15,5$ \\
& Invierno & $148,8 \pm 94,8$ \\
& Primavera & $130,6 \pm 117,0$ \\
\hline
\end{tabular}

según el uso estándar (APHA et al., 1981): pH, RSF, $\mathrm{Fe}, \mathrm{Mn}, \mathrm{Cu}, \mathrm{As}$ total, $\mathrm{Fl}$ y sulfato. Para la descripción y caracterización de la variabilidad altitudinal se usaron datos obtenidos en estaciones instaladas a $3.920,3.940$ y $3.970 \mathrm{~m} \mathrm{~s} \mathrm{n} \mathrm{m}$, registrados en el verano de 1999. Para este objetivo los parámetros físicos examinados fueron conductividad eléctrica, dureza total, sólidos en suspensión y sólidos totales $\left(105^{\circ} \mathrm{C}\right)$; a su vez, los parámetros hidroquímicos examinados fueron $\mathrm{pH}$, acidez titulable, alcalinidad, $\mathrm{Ag}, \mathrm{Al}$, As total, $\mathrm{Ca}, \mathrm{Cd}, \mathrm{CN}$ total, $\mathrm{Cu}, \mathrm{Cr}, \mathrm{Fe}, \mathrm{Fl}$, $\mathrm{Hg}, \mathrm{K}, \mathrm{Mg}, \mathrm{Mn}, \mathrm{Ni}, \mathrm{Pb}, \mathrm{Zn}$, bicarbonato, fosfato, sulfato, nitrato y nitrito. Debido al número de parámetros medidos, no hubo réplica altitudinal de los análisis químicos.

Una empresa contratista especializada (SITAC, 2001) realizó la toma de muestras y los análisis respectivos, según las normas y técnicas estándares establecidas para ello y descritas en APHA et al. (1981), bajo la supervisión de CONAMA y DGA.

\section{Resultados y Discusión}

\section{Dinámica fluviométrica del estero Tambo}

Ya que durante el período analizado no se observaron flujos nulos, se infiere que el régimen del estero Tambo es de tipo permanente, aunque muy variable tanto mensual como anualmente (Tabla 1, Figura 1). El promedio anual del flujo superficial varió entre 40 y 300 1/s (años 1995 y 1983, respectivamente); con variabilidad más alta a caudal mayor. Los caudales más bajos mostraron

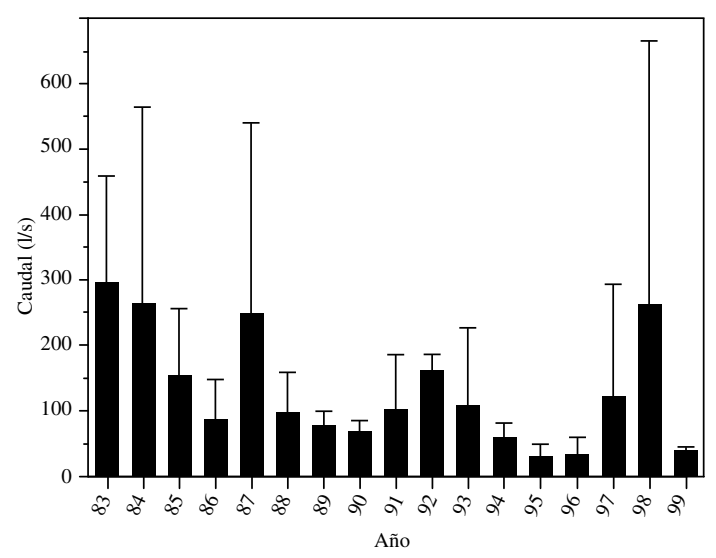

Figura 1. Variabilidad temporal del promedio del caudal superficial (1/s, \pm DE) del estero Tambo. Altitud: $3940 \mathrm{~m} \mathrm{~s} \mathrm{n} \mathrm{m}$. Serie de tiempo: 1983-1999. 
correspondencia con la precipitación registrada; esta correspondencia no se evidenció claramente en el caso de los caudales más altos (Figura 1). El promedio mensual osciló en torno a $\sim 131$ 1/s. Se mantuvo dentro de un margen relativamente estrecho entre enero $(22,4 \mathrm{l} / \mathrm{s})$ y octubre $(84,3 \mathrm{l} / \mathrm{s})$, para aumentar bruscamente en noviembre $(151,7 \mathrm{l} / \mathrm{s})$ y diciembre $(210,5 \mathrm{l} / \mathrm{s})$. Primavera y verano fueron las estaciones con los flujos más altos; lo contrario fue registrado en otoño e invierno (Tabla 1). Este comportamiento es consistente con observaciones realizadas en otras hoyas hidrográficas del desierto transicional de Chile (Vicuña et al., 2011). La correspondencia entre la precipitación observada durante el período estudiado y el caudal superficial sugiere que el caudal mínimo se obtendría con precipitaciones inferiores al $40 \%$ del promedio anual (es decir, $<81 \mathrm{~mm} /$ año). Según Zavala (2006, 2009), en consideración a que los caudales de deshielo y de acumulación de nieve tienden a ser similares durante los años secos, los caudales medios mensuales del estero Tambo asociados a probabilidades de excedencia alta $(>70 \%)$ no difieren mayormente entre sí. Esta situación ocurre porque los caudales de los años secos se originan principalmente en flujos bajos (p. e., aporte de acuíferos). En oposición, los caudales medios mensuales asociados a probabilidades de excedencia baja $(<40 \%)$ (p. e., años ENSO) presentan diferencias importantes entre caudales de deshielo y caudales de acumulación de nieve. Esto debido a la contribución del deshielo, fenómeno que ocurre en estas latitudes más intensamente durante el período octubre-marzo que durante el período abril-septiembre.

\section{Dinámica de las características físicas y químicas del flujo superficial del estero Tambo}

Altitudinalmente algunas características del agua superficial del estero Tambo difirieron marcadamente. Por ejemplo, la conductividad eléctrica, dureza y acidez titulable y los niveles de $\mathrm{Ca}, \mathrm{Fe}, \mathrm{Fl}$ y $\mathrm{Mn}$ fueron más altos a los $3.920 \mathrm{~m} \mathrm{~s} \mathrm{n} \mathrm{m;} \mathrm{por} \mathrm{el} \mathrm{contrario,}$ a esa altitud se registró un valor anormalmente bajo de $\mathrm{pH}$ (Tabla 2). Lo anterior probablemente debido a la contribución de las quebradas aportantes ubicadas a mayor altitud y/o a la actividad minera realizada en las cercanías (Oyarzún et al., 2006). En sus aspectos más destacados, el análisis de la serie 1982-1999 mostró valores anuales inferiores a 7,0 para el caso del pH; el nivel de RSF tomó
Tabla 2. Variabilidad altitudinal de parámetros físicos y químicos del agua superficial del estero Tambo. Cifras en $\mathrm{mg} / \mathrm{l}$; excepto $\mathrm{pH}$ y conductividad ( $\mu \mathrm{mhos} / \mathrm{cm}$, a $25^{\circ} \mathrm{C}$ ).

\begin{tabular}{|c|c|c|c|}
\hline \multirow[b]{2}{*}{ Parámetro } & \multicolumn{3}{|c|}{ Altitud (m s n m) } \\
\hline & 3.920 & 3.940 & 3.970 \\
\hline \multicolumn{4}{|l|}{ Físicos } \\
\hline Conductividad & $1.540,0$ & 439,6 & 436,8 \\
\hline Dureza & 535,4 & 122,3 & 176,7 \\
\hline Sólidos en suspensión & 48,1 & 28,6 & 79,8 \\
\hline Sólidos totales $\left(\mathrm{a} 105^{\circ} \mathrm{C}\right)$ & ND & 370,0 & 507,2 \\
\hline \multicolumn{4}{|l|}{ Químicos } \\
\hline $\mathrm{pH}$ & 3,7 & 7,2 & 7,2 \\
\hline Acidez (titulable) & 198,7 & 4,6 & 6,9 \\
\hline Alcalinidad & ND & 22,6 & 22,0 \\
\hline $\mathrm{Ag}$ & 0,02 & 0,02 & 0,02 \\
\hline $\mathrm{Al}$ & 0,5 & 0,4 & 2,2 \\
\hline As total & 0,04 & 0,1 & 0,1 \\
\hline Bicarbonato & ND & 27,2 & 26,8 \\
\hline $\mathrm{Ca}$ & 111,6 & 29,3 & 27,9 \\
\hline $\mathrm{Cd}$ & 0,01 & $<0,01$ & $<0,01$ \\
\hline $\mathrm{CN}$ (total) & 0,1 & 0,1 & 0,1 \\
\hline $\mathrm{Cu}$ & 0,03 & 0,1 & 0,03 \\
\hline $\mathrm{Cr}$ & 0,02 & $<0,01$ & $<0,01$ \\
\hline $\mathrm{Fe}$ & 64,5 & 0,5 & 0,4 \\
\hline $\mathrm{Fl}$ & 8,7 & 0,6 & 0,7 \\
\hline Fosfato & 1,6 & 1,6 & 3,4 \\
\hline $\mathrm{Hg}$ & $<0,01$ & $<0,01$ & $<0,01$ \\
\hline $\mathrm{K}$ & ND & 4,9 & ND \\
\hline $\mathrm{Mg}$ & ND & 8,3 & ND \\
\hline $\mathrm{Mn}$ & 12,7 & 0,2 & 0,2 \\
\hline $\mathrm{Ni}$ & 0,1 & 0,1 & $<0,01$ \\
\hline Nitrato & 0,3 & 0,3 & 13,6 \\
\hline Nitrito & 0,02 & 0,01 & 0,01 \\
\hline $\mathrm{Pb}$ & 0,02 & 0,02 & 0,05 \\
\hline Sulfato & ND & 900,0 & ND \\
\hline
\end{tabular}

ND: no determinado.

valores entre $1.085,5$ y $2.196,7 \mathrm{mg} / \mathrm{l}$ y el nivel de sulfato varió entre 477,7 y $1173,7 \mathrm{mg} / \mathrm{l}$. El nivel anual de $\mathrm{Fe}$ mostró valores erráticos (muy altos) en otoño $(84,5 \mathrm{mg} / \mathrm{l})$ e invierno $(53,0 \mathrm{mg} / \mathrm{l})$. Los otros componentes mostraron rangos de valores más estrechos (Tabla 3). El comportamiento anual y mensual de los parámetros examinados se grafica en las Figs. 2 a 9. En general, la variabilidad asociada a los promedios mensuales fue alta, particularmente en los casos de RSF (Figura 2a), pH (Figura 3a), Fe (Figura 4a) y As total (Figura 8a). Una situación similar se registró con los promedios anuales, particularmente en el caso del Fe (Figura 4b). Esta variabilidad impidió detectar diferencias asociadas a años o a estaciones. Valores anómalos se registraron con el pH (Figura 3b, año 1990, valor muy bajo en comparación al resto de la serie) y con el Fe 
Tabla 3. Rangos estacionales y anuales de parámetros químicos del estero Tambo. Altitud: 3.940 m s n m. Serie de tiempo: 1982-1999. Cifras en mg/l; excepto $\mathrm{pH}$.

\begin{tabular}{|c|c|c|}
\hline Parámetro & Estación & Rango \\
\hline \multirow[t]{5}{*}{$\overline{\mathrm{pH}}$} & Verano & $4,8-7,3$ \\
\hline & Otoño & $4,7-6,5$ \\
\hline & Invierno & $4,7-6,7$ \\
\hline & Primavera & $4,7-7,2$ \\
\hline & Anual & $4,8-6,8$ \\
\hline \multirow[t]{5}{*}{ RSF } & Verano & $824,7-2015,0$ \\
\hline & Otoño & $1066,7-2250,0$ \\
\hline & Invierno & $1253,0-2306,7$ \\
\hline & Primavera & $721,3-2516,7$ \\
\hline & Anual & $1085,5-2196,7$ \\
\hline \multirow[t]{5}{*}{$\mathrm{Fe}$} & Verano & $5,9-29,0$ \\
\hline & Otoño & $6,5-84,5$ \\
\hline & Invierno & $5,3-53,0$ \\
\hline & Primavera & $37,4-7,2$ \\
\hline & Anual & $6,7-25,6$ \\
\hline \multirow[t]{5}{*}{ Mn } & Verano & $1,8-4,7$ \\
\hline & Otoño & $2,7-6,1$ \\
\hline & Invierno & $2,5-7,0$ \\
\hline & Primavera & $1,7-4,5$ \\
\hline & Anual & $2,8-4,5$ \\
\hline \multirow[t]{5}{*}{$\mathrm{Cu}$} & Verano & $0,1-2,0$ \\
\hline & Otoño & $0,1-1,5$ \\
\hline & Invierno & $0,1-0,5$ \\
\hline & Primavera & $0,1-0,3$ \\
\hline & Anual & $0,1-0,6$ \\
\hline \multirow[t]{5}{*}{ As total } & Verano & $0,03-0,6$ \\
\hline & Otoño & $0,2-0,8$ \\
\hline & Invierno & $0,3-0,7$ \\
\hline & Primavera & $0,3-1,0$ \\
\hline & Anual & $0,3-0,7$ \\
\hline \multirow[t]{5}{*}{$\mathrm{Fl}$} & Verano & $0,6-3,0$ \\
\hline & Otoño & $0,8-2,8$ \\
\hline & Invierno & $0,5-2,2$ \\
\hline & Primavera & $0,3-1,6$ \\
\hline & Anual & $0,7-1,7$ \\
\hline \multirow[t]{5}{*}{ sulfato } & Verano & $443,0-1065,0$ \\
\hline & Otoño & $?-1236,7$ \\
\hline & Invierno & $508,9-1292,5$ \\
\hline & Primavera & $416,3-1310,0$ \\
\hline & Anual & $477,7-1173,7$ \\
\hline
\end{tabular}

(Figura 4b, situación similar a la anterior) y con el Cu (Figura 6b, años 1987 y 1989, valores muy altos en relación al resto de la serie).

\section{Tipificación del agua superficial del estero Tambo}

Las aguas del estero Tambo corresponden a aguas muy duras (3.920 $\mathrm{m} \mathrm{s} \mathrm{n} \mathrm{m)} \mathrm{y} \mathrm{a} \mathrm{duras} \mathrm{a}$ moderadamente duras para los pisos superiores. El contenido de sólidos suspendidos supera el valor

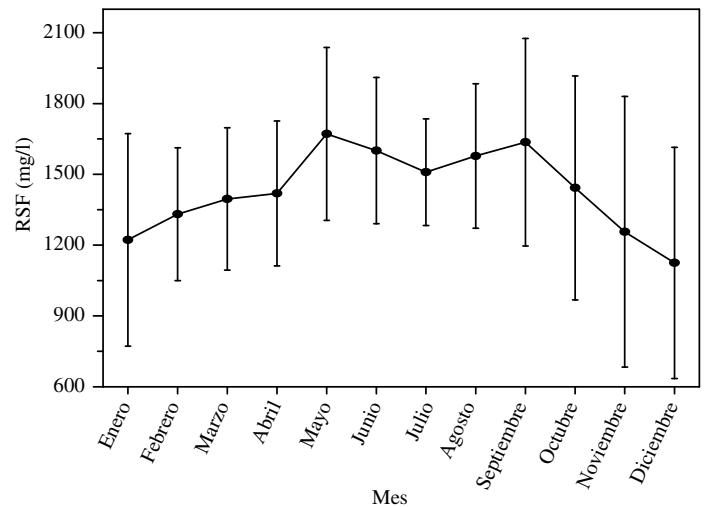

Figura 2a. Variabilidad mensual del nivel promedio de RSF $(\mathrm{mg} / \mathrm{l}, \pm \mathrm{DE})$ en el agua superficial del estero Tambo. Serie de tiempo: 1982-1999.

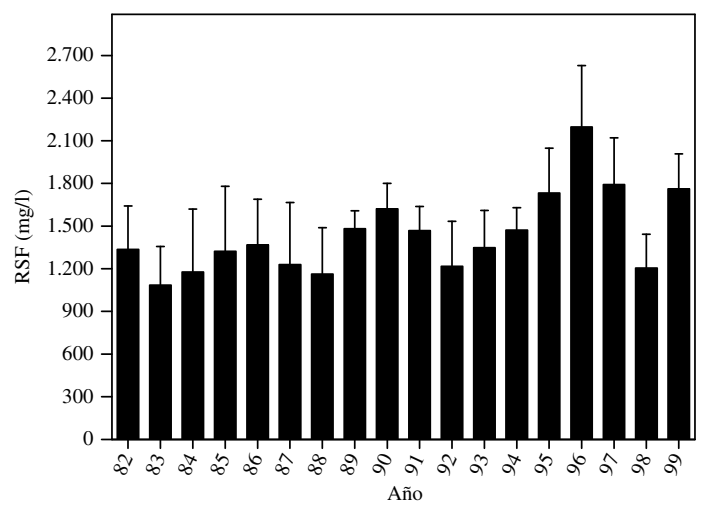

Figura 2b. Variabilidad anual del nivel promedio de RSF (mg/l, \pm DE) en el agua superficial del estero Tambo. Serie de tiempo: como en Figura 2a.

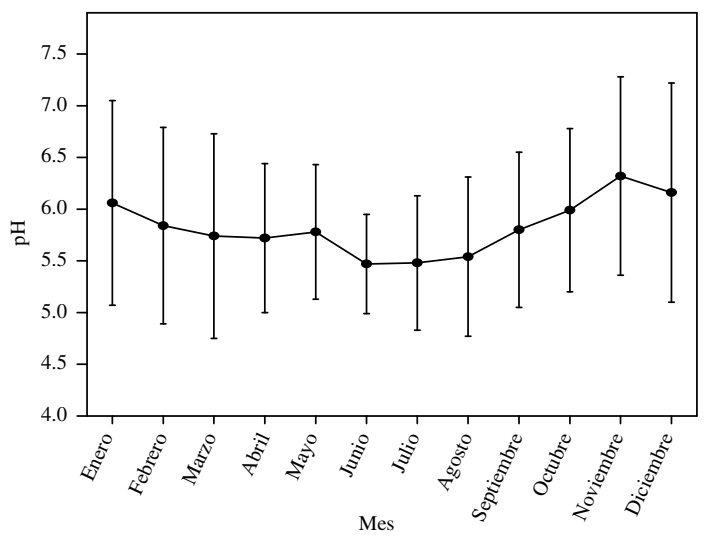

Figura 3a.Variabilidad mensual del $\mathrm{pH}$ promedio $( \pm \mathrm{DE})$ del agua superficial del estero Tambo. Serie de tiempo: como en Figura 2a.

límite superior establecido para la vida acuática en la normativa estadounidense (Chapman, 1996), encontrándose en el rango inferior del tipo aguas 


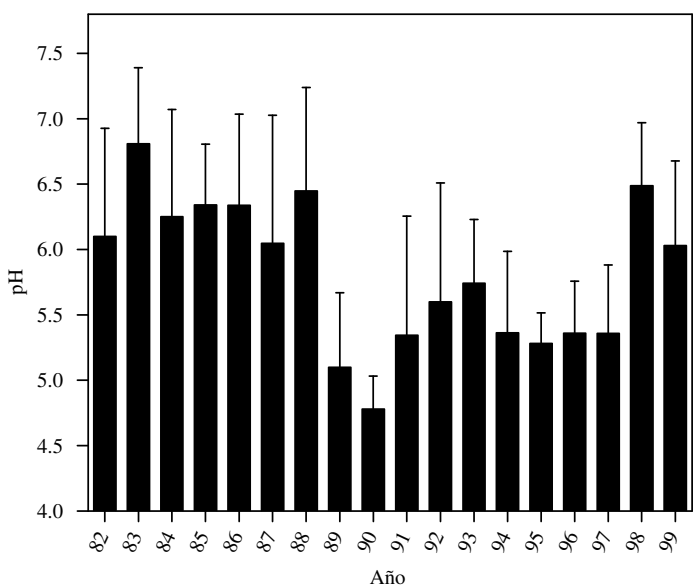

Figura 3b. Variabilidad anual del $\mathrm{pH}$ promedio $( \pm \mathrm{DE})$ del agua superficial del estero Tambo. Serie de tiempo: como en Figura 2a.

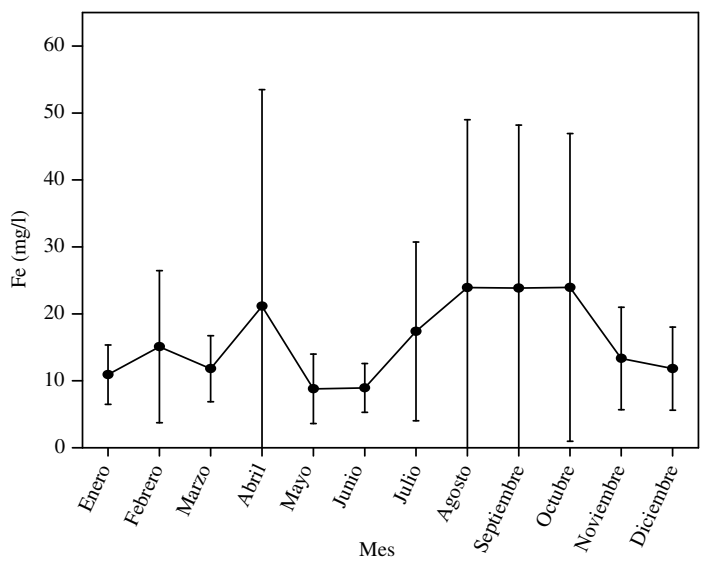

Fig.4a.Variabilidad mensual del nivel promedio de $\mathrm{Fe}(\mathrm{mg} / \mathrm{l}, \pm$ DE) en el agua superficial del estero Tambo. Serie de tiempo: como en Figura 2a.

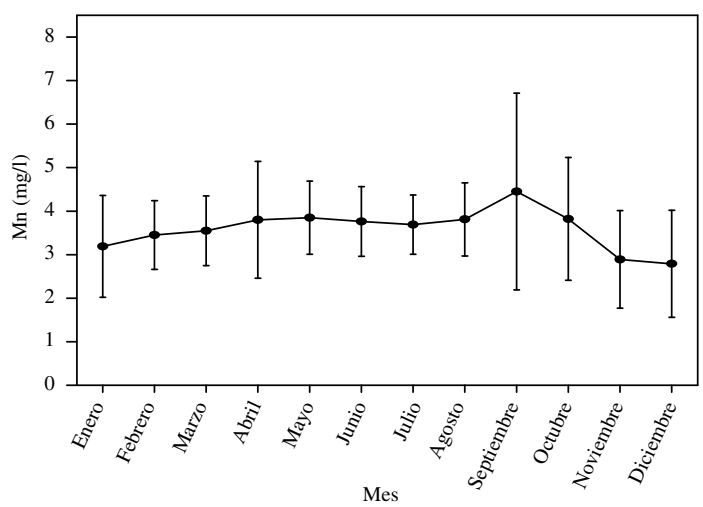

Figura 5a.Variabilidad mensual del nivel promedio de $\mathrm{Mn}(\mathrm{mg} / \mathrm{l}$, $\pm \mathrm{DE}$ ) en el agua superficial del estero Tambo. Serie de tiempo: como en Figura 2a. salobres (Arribas et al., 2009). Desde el punto de vista de la clase de hábitat acuático (CONAMA, 2000), el estero Tambo clasifica en clase 1 (estación $3.940 \mathrm{~m} \mathrm{~s} \mathrm{n} \mathrm{m}$ ) y clase 3 (estaciones $3.970 \mathrm{~m} \mathrm{~s} \mathrm{n} \mathrm{m}$ y $3.940 \mathrm{~m} \mathrm{~s} \mathrm{n} \mathrm{m}$ ). Para RSF, las aguas corresponden a las clases 0 y 1 (CONAMA, 2000). Considerando como criterio a la conductividad eléctrica, las aguas de las secciones superiores del tramo estudiado se comportan como aguas dulces, con valores $<1.500$ $\mu \mathrm{S} / \mathrm{cm}$ y ligeramente subsalinas en la sección inferior $(\sim 1.500 \mu \mathrm{S} / \mathrm{cm})$. Los valores de $\mathrm{pH}$ se hallan dentro del rango informado para aguas naturales $(6,5-8,0)$. $\mathrm{Si}$ se considera el valor promedio del $\mathrm{pH}$, la clase de calidad de agua del estero Tambo es 4 (CONAMA, 2000), concordando con lo reportado por DGA (2004) para el nivel altitudinal de $2.520 \mathrm{~m} \mathrm{~s} \mathrm{n} \mathrm{m.}$

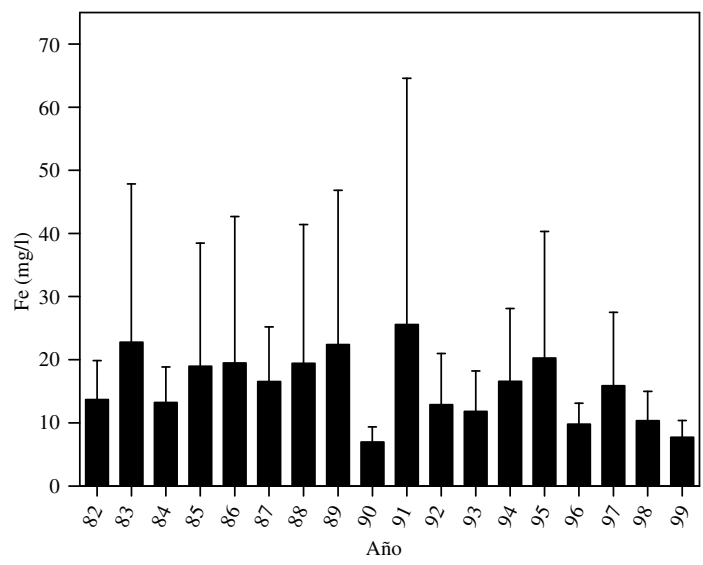

Figura 4b.Variabilidad anual de la concentración promedio de $\mathrm{Fe}(\mathrm{mg} / \mathrm{l}, \pm \mathrm{DE})$ en el agua superficial del estero Tambo. Serie de tiempo: como en Figura 2a.

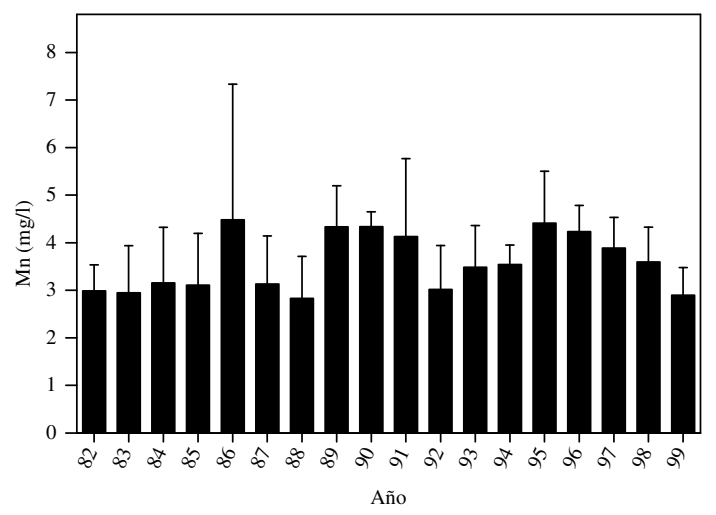

Figura 5b.Variabilidad anual de la concentración promedio de $\mathrm{Mn}(\mathrm{mg} / \mathrm{l}, \pm \mathrm{DE})$ en el agua superficial del estero Tambo. Serie de tiempo: como en Figura 2a. 


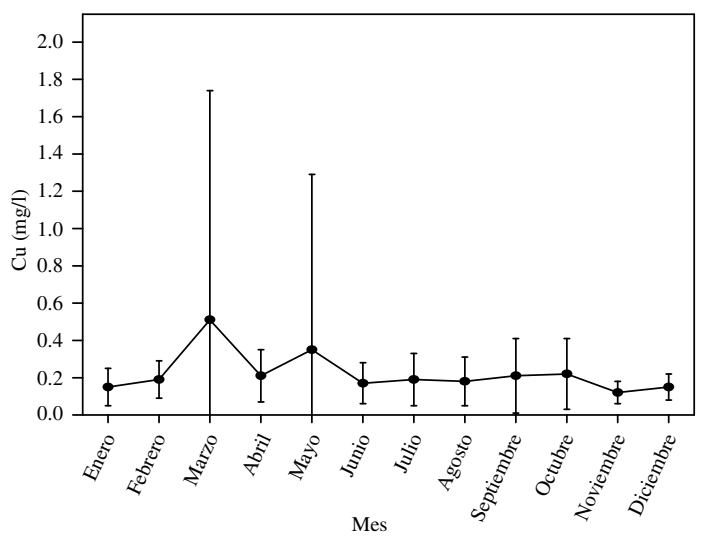

Figura 6a Variabilidad mensual del nivel promedio de $\mathrm{Cu}(\mathrm{mg} / \mathrm{l}$, \pm DE) en el agua superficial del estero Tambo. Serie de tiempo: como en Figura 2a.

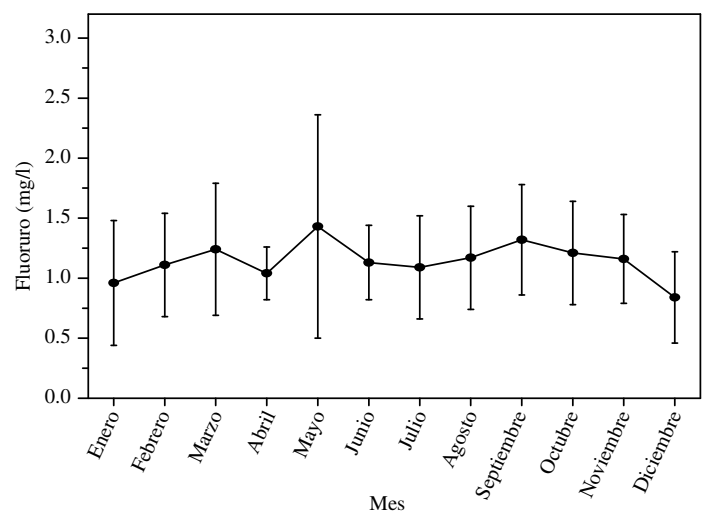

Figura 7a. Variabilidad mensual del nivel promedio de As (mg/l, \pm DE) en el agua superficial del estero Tambo. Serie de tiempo: como en Figura 2a.

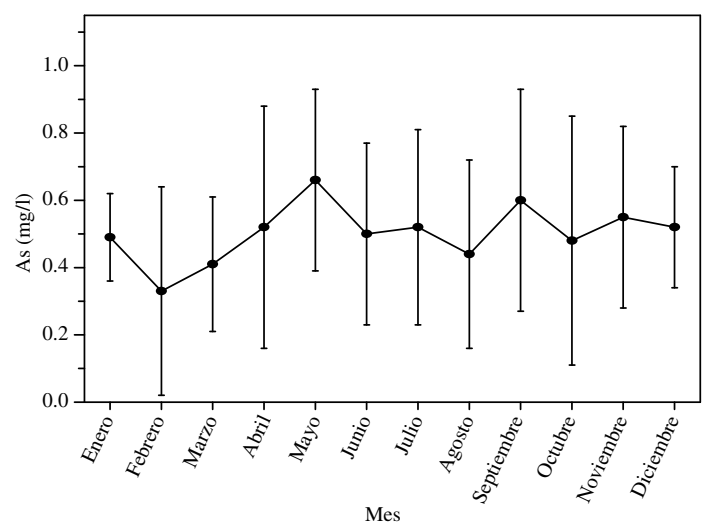

Figura 8a.Variabilidad mensual del nivel promedio de fluoruro (mg/l, \pm DE) en el agua superficial del estero Tambo. Serie de tiempo: como en Figura 2a.

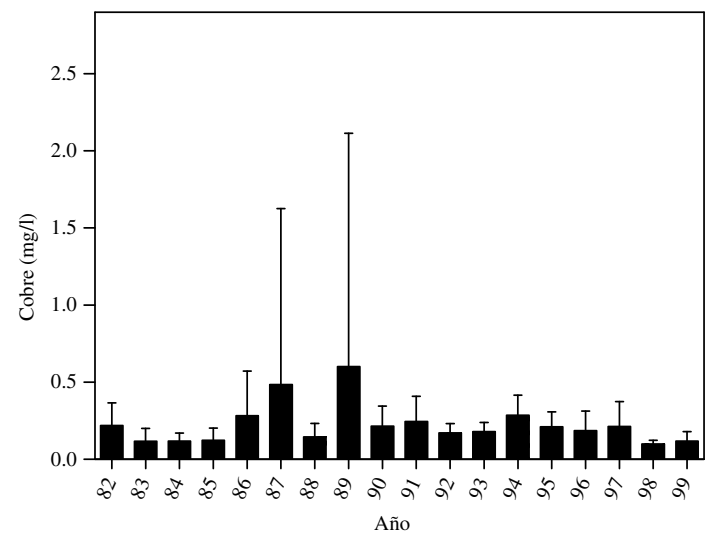

Figura 6b. Variabilidad anual del contenido promedio de $\mathrm{Cu}$ $(\mathrm{mg} / \mathrm{l}, \pm \mathrm{DE})$ en el agua superficial del estero Tambo. Serie de tiempo: como en Figura 2a.

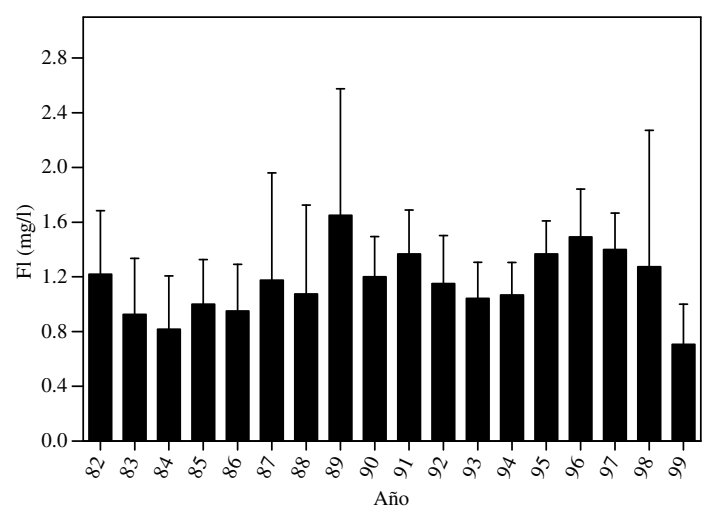

Figura 7b.Variabilidad anual del nivel promedio de As total $(\mathrm{mg} / \mathrm{l}, \pm \mathrm{DE})$ en el agua superficial del estero Tambo. Serie de tiempo: como en Figura 2a.

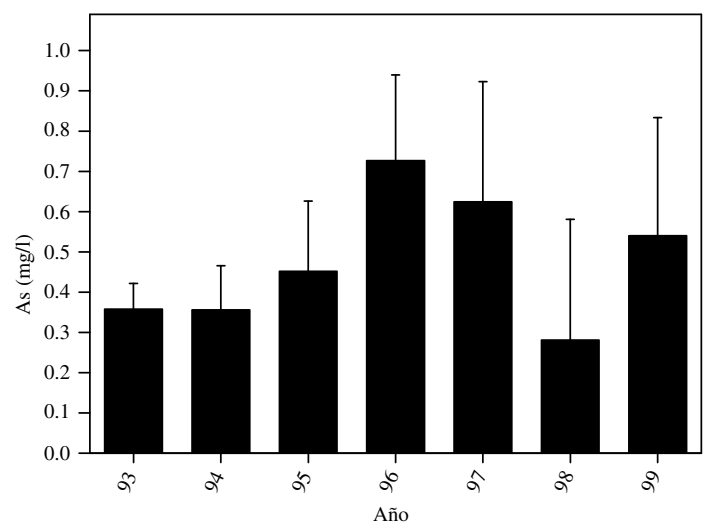

Figura 8b.Variabilidad anual de la concentración promedio de $\mathrm{Fl}(\mathrm{mg} / \mathrm{l}, \pm \mathrm{DE})$ en el agua superficial del estero Tambo. Serie de tiempo: como en Figura 2a. 


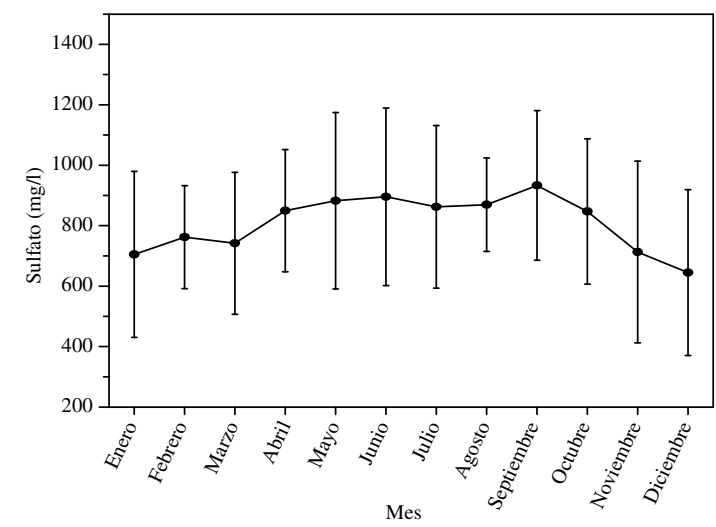

Figura 9a.Variabilidad mensual del nivel promedio de sulfato $(\mathrm{mg} / \mathrm{l}, \pm \mathrm{DE})$ en el agua superficial del estero Tambo. Serie de tiempo: como en Figura 2a.

La alcalinidad promedio es ligeramente superior al límite mínimo establecido en la NCh 1333-78 para la vida acuática (INN, 1978). Respecto del contenido de sulfato, las aguas estudiadas se encuentran en las clases 2 y 3 (CONAMA, 2000). El índice $\mathrm{Mg} / \mathrm{Ca}$ fue $\sim 0,28$, un valor algo inferior al reportado para aguas continentales (Chapman, 1996). Si se considera el nivel de Fl, las aguas del estero Tambo son categoría 1 y 2 (CONAMA, 2000). El nivel de K no presenta situación especial. El promedio mensual de Fe superó ampliamente lo establecido para aguas clase 3 (CONAMA, 2000). Los componentes trazas que se encontraron en concentraciones $>0,1 \mathrm{mg} / \mathrm{l}$ (Chapman, 1996) fueron $\mathrm{Al}$, As total, Cu y Mn. A continuación, en paréntesis, se indica la clase de hábitat por constituyente traza, según CONAMA (2000): Al y Pb (clase 2), $\mathrm{Zn}$ (clases 1 y 2), $\mathrm{As}, \mathrm{Cu}, \mathrm{Mn}$ y $\mathrm{CN}$ (superan la norma). Componentes con promedios $<0,1 \mathrm{mg} / \mathrm{l}$ fueron $\mathrm{Ni}, \mathrm{Ag}, \mathrm{Cd}, \mathrm{Cr}$ y $\mathrm{Hg}$. En paréntesis se indica la clase de hábitat por constituyente traza $<0,1 \mathrm{mg} / \mathrm{l}$ según CONAMA (2000): Ni (entre clases 1 y 2), $\mathrm{Cd}$ (clase 2), $\mathrm{Cr}$ (entre clases 1 y 2 ) y $\mathrm{Hg}$ (supera la norma). Los elevados niveles de As total han sido declarados naturales (DGA, 2004), a pesar de cierta controversia al respecto (Oyarzún et al., 2006). Las características arriba descritas explican la pobreza de la biota acuática registrada en sus aguas (Cepeda et al., 2006).

La variabilidad asociada a los niveles medios de los parámetros estudiados en este trabajo concuerda con lo observado en ecosistemas fluviales de climas áridos de otras latitudes, argumentándose como causa principal de ella al patrón pluviométrico

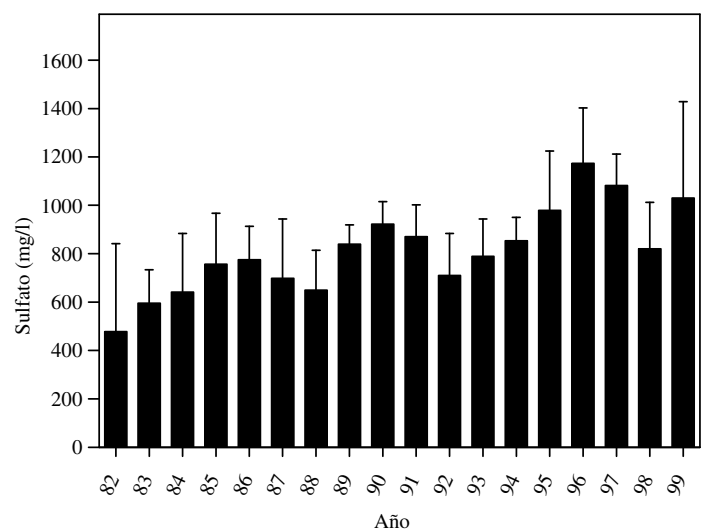

Figura 9b.Variabilidad anual de la concentración de sulfato promedio $(\mathrm{mg} / \mathrm{l}, \pm \mathrm{DE})$ en el agua superficial del estero Tambo. Serie de tiempo: como en Figura 2a.

(Vidal-Abarca et al., 2004; Millán et al., 2011). Por otro lado, para el caso de ecosistemas de montaña, algunos autores (p. e., Queirolo et al., 2000; Pittaluga y Suvires, 2006) han enfatizado la importancia de las características mineralógicas del sustrato por donde drena el agua; influencia que ocurriría en el área de estudio, según ha sido documentado por Strauch et al. (2006).

En el norte de Chile, por causas tanto naturales como antrópicas, el agua está siendo un recurso cada vez más escaso. En esta región las cuencas proveedoras de agua son andinas. Por ejemplo, $>85 \%$ del agua que recibe el piso costero de la hoya hidrográfica del río Elqui corresponde a nieve acumulada en los pisos andinos (Zavala, 2009). Además de proveer agua, estas cuencas acogen a sistemas naturales que, en un medio árido, constituyen islas (p. e., bofedales, vegas) que concentran recursos biológicos y patrimoniales de interés para diferentes actores sociales (Osorio et al., 2006). Además de la presencia de agua, estas cuencas y la montaña circundante contienen recursos minerales que las hacen atractivas para una minería intensiva (Oyarzún et al., 2006). Todo lo anterior contribuye a generar un escenario de conflictos ambientales potenciales cuya atención y toma de decisiones demandan poseer el adecuado conocimiento de ellas. Este trabajo estuvo orientado en esa dirección.

\section{Conclusiones}

El estero Tambo resultó ser un cuerpo de agua permanente. En sus aguas se registró la presencia de 
metales, componentes no metálicos inorgánicos y elementos traza propios del sustrato geológico de las montañas andinas circundantes. Las características físicas y químicas medidas mostraron una elevada variabilidad temporal en torno a los niveles promedio, aparentemente en concordancia con el patrón pluviométrico del área, según lo observado en ecosistemas fluviales de climas áridos y semiáridos de otras latitudes. Algunos parámetros mostraron una conducta errática, probablemente debida a la influencia de la actividad minera que se realiza en las inmediaciones del estero. No se detectaron patrones estaciones en el comportamiento de los parámetros monitoreados, como era esperado según las características del clima mediterráneo imperante en la zona. Contribuyeron a ello, aparentemente, tanto las fluctuaciones naturales de las características físicas y químicas del cuerpo de agua como los impactos sobre estas que recibe de la actividad minera circundante.

\section{Agradecimientos}

Esta publicación fue financiada por la Dirección de Investigación de la Universidad de La Serena (La Serena, Chile) por medio del proyecto CD01020795 a J. Cepeda P. Agradecemos el acceso a la base de datos del programa de monitoreo de calidad de aguas de la ex-Compañía Minera El Indio (alta montaña del valle de Elqui, Chile).

\section{Literatura Citada}

APHA; AWWA; WPCF.

1981. Standard Methods for the Examination of Water and Wastewater.American Public Health Association American (APHA), American Water Works Association (AWWA) and Water Pollution Control Federation (WPCF). 16th edition. Washington D.C.

Arribas, P.; Gutiérrez-Cánovas, C.; Abellán, P.; Sánchez-Fernández,

D.; Picazo, F.; Velasco, J.; Millán, A.

2009. Tipificación de los ríos salinos ibéricos. Ecosistemas, 18: 1-13.

Cepeda-Pizarro, J.

2013. Variabilidad temporal de algunos parámetros físicos e hidroquímicos de un prado húmedo altoandino del nortecentro de Chile. Idesia, 31: 89-97.

Cepeda P., J.; Novoa, J.

2006. La cordillera alto andina del Valle del Elqui. En: Geoecología de los Andes desérticos. La alta montaña del valle del Elqui. Cepeda P., J. (ed.). Ediciones Universidad de La Serena. La Serena, Chile, pp. 39-66.

Cepeda P., J.; Squeo P., F.; Cortés M., A.; Oyarzún M., J.;

Zavala Z., H.

2006. La biota del humedal Tambo-Puquíos. En: Geoecología de los Andes desérticos. La alta montaña del valle del Elqui. Cepeda P., J. (ed.). Ediciones Universidad de La Serena. La Serena, Chile, pp. 243-283.

CONAMA.

2000. Guía para el establecimiento de las normas secundarias de calidad ambiental para aguas continentales superficiales y marinas (Informe Técnico). Comisión Nacional del Medio Ambiente. Gobierno de Chile. Santiago, Chile.

Cooper, D.J.; Wolf, E.C.; Colson, C.; Vering, W.; Granda, A.; Meyer, M.

2010. Alpine peatlands of the Andes, Cajamarca, Peru. Arctic, Antarctic and Alpine Research, 42: 19-33.

Chapman, D. (ed.).

1996. Water quality assessments. A guide to use of biota, sediments and water in environmental monitoring. $2^{\text {nd }}$ Edition. UNESCO/WHO/UNEP. E \& FN Spon, an imprint of Chapman and Hall. Cambridge UniversityPress. Great Britain.

DGA.

2004. Diagnóstico y clasificación de los cuerpos de agua según objetivos de calidad. Cuenca del río Elqui (Informe Técnico). Dirección General de Aguas. Ministerio de Obras Públicas. Gobierno de Chile. Santiago, Chile.

Ginocchio, R.; Hepp, J.; Bustamante, E.; Silva, Y.; De La Fuente, L.M.; Casale, J.F.; De La Harpe, J.P.; Urrestarazu, P.; Anic,V.; Montenegro, G.

2008. Importance of water quality on plant abundance and diversity in high-alpine meadows of the Yerba Loca Natural Sanctuary at the Andes of north-central Chile. Revista Chilena de Historia Natural, 81: 469-488.

INN

1978. Requisitos de calidad de agua para diferentes usos (Informe Técnico). Norma chilena oficial (NCH 1333, oficio 1978, modificada 1987). Normas chilenas. Catálogo 2012. Instituto Nacional de Normalización (INN).Gobierno de Chile. Santiago, Chile.

Millán, A.; Velasco, J.; Gutiérrez-Cánovas, C.; Arribas, P.; Picazo,

F.; Sánchez-Fernández, D.; Abellán, P.

2011. Mediterranean saline streams in the southeast Spain: What do we know? Journal of Arid Environments, 75: 1352-1359.

Pittaluga, M.A.; Suvires, G.M.

2006. Chemical composition and water quality of the Castaño river, Calingasta, San Juan Province, Argentina. Multequina, 15: $17-26$.

Osorio B., R.; Cabezas C., R.; Reyes S., H.; Álvarez L., P.; Koné, T. 2006. Humedales altoandinos de la IV Región. En: Geoecología de los Andes desérticos. La alta montaña del valle del Elqui. J. Cepeda P. (ed.). Ediciones Universidad de La Serena. La Serena, Chile, pp. 153-239.

Oyarzún, R.; Guevara, S.; Oyarzún, J.; Lillo, J.; Maturana, H.; Higueras, $\mathrm{P}$.

2006. The As-contaminated Elqui river basin: a long lasting perspective (1975-1995) covering the initiation and development of Au-Cu-As mining in the high Andes of 
northern Chile. Environmental Geochemistry and Health, 28: 431-443.

Queirolo, F.; Stegen, S.; Mondaca, J.; Cortés, R.; Rojas, R.; Contreras, C.; Muñoz, L.; Schwuger, M.J.; Ostapczuk, P.

2000. Total arsenic, lead. Cadmium, copper, and zinc in some salt rivers in the northern Andes of Antofagasta, Chile. Science of the Total Environment, 255: 85-95.

SITAC.

2001. Programa de control de calidad de agua. Cuenca del Río Elqui. IV Región de Coquimbo. Compañía Minera El Indio (Informes Técnicos Anuales período 1983-2000). Santiago, Chile.

Squeo, F.; Warner, B.G.; Aravena, R.; Espinoza, D.

2006. Bofedales: high altitude peatlands of the central Andes. Revista Chilena de Historia Natural 79, 245-255.

Strauch, G.; Oyarzún, J.; Fiebig-Wittmaack, M.; González, E.; Weise, S.M.

2006. Contributions of the different water sources to the Elqui river runoff (northern Chile) evaluated by $\mathrm{H} / \mathrm{O}$ isotopes.
Isotopes in Environmental and Health Studies, 42: 303-322.

Vidal-Abarca, M.R.; Gómez, R.; Suárez, M.L.

2004. Los ríos de las regiones semiáridas. Ecosistemas, 13: $16-28$

Vicuña, S.; Garreaud, R.D.; McPhee, J.

2011. Climate change impacts on the hydrology of a snowmelt driven basin in semiarid Chile. Climatic Change, 105: 469-488.

Zavala, $\mathrm{H}$.

2006. Hidrología del humedal Tambo-Puquíos.En: Geoecología de los Andes desérticos. La alta montaña del valle del Elqui. J. Cepeda P. (ed.). Ediciones Universidad de La Serena. La Serena, Chile, pp. 285-322.

Zavala, $\mathrm{H}$.

2009. Hidrología de la cuenca del río Elqui. En: Los sistemas naturales de la cuenca del río Elqui (Región de Coquimbo, Chile): vulnerabilidad y cambio del clima. J. Cepeda P. (ed.): Ediciones Universidad de La Serena La Serena, Chile, pp. 59-156. 
